\title{
Novel Modeling of the Work-Hardening Curve
}

\author{
Zdzisław Pluta, Tadeusz Hryniewicz* \\ Koszalin University of Technology, Raclawicka 15-17, PL 75-620 Koszalin, Poland \\ *E-Mail address: Tadeusz.Hryniewicz@tu.koszalin.pl
}

\begin{abstract}
„Scientific explanation does not rely on getting from the complexity to simplicity, but on substituting the less understood complexity by more comprehensible."
\end{abstract}

Lévi-Strauss

\begin{abstract}
The paper aims at a critical analysis of the existent description of plastic strengthening/ workhardening curve. The adequate description of the curve, having a physical sense, has been proposed. The description begins from the very source which is the primary, general differential equation. That source was used to formulate the characteristics of the studied system. Then the analytical solution of the dependence between the yield stress and the relative deformation is provided. The description of the work-hardening curve has been developed to further form a proper dependence of the yield force on the absolute yield strain. Other functional characteristics, namely the coefficient of the plastic rigidity, yield work, and the plastic potentials, have also been formed.
\end{abstract}

Keywords: Hardening curve; Yield stress; Relative deformation; Relative deformation constant; Absolute strain constant; Stress potential fields; Strain potential fields; Yield force; Plastic rigidity; Plastic energy; Yield work

\section{INTRODUCTION}

There is an increasing tendency observed to simplify the descriptions of natural systems, relying on the creating of models being some approximation of the systems. Physical phenomena are described by various mathematical functions. These functions are often represented by lines connecting experimental points characteristic with a dispersion. The statistics calls the lines as the regression lines running through the point patterns (statistic materials) so that the best fitting of both elements, continuous and dispersive, is achieved.

Thus created model, an empirical statistic-experimental creation/piece of work, usually does not possess a physical meaning and corresponds only to the planned variability interval of the determined magnitudes. Therefore such a model does not cover the whole considered phenomenon, but to its limited part only.

There are some efforts to foresee the course of a studied magnitude (dependent variable) beyond the experimental range, called the extrapolation; however, these activities do not have an adequate character and lead sometimes behind the limits of the phenomenon area, up to the infinity. In these actions the notion of the initial conditions is often dispersed; the conditions 
are often assumed as the beginning of measurement of a determined magnitude, whereas they should refer to the beginning of the very phenomenon. Another quest is a criticless and improper description of the phenomenon in its initial period, in the neighbourhood of zero.

The authors of this work have described many different parts of the macro-reality. That way, regarding the quantum nature, the tool life under cut on the main velocity, concerning the rotation of the tool or workpiece [1], has been described. In the frameworks of these activities the essence of the initial conditions of the described process or phenomenon, has been explained. In the works $[2,3]$ that essence was referred to the process of surface finishing using elastic abrasive wheels, with the fundamentals provided in the monograph [4].

This work has been focused onto another system. It is about the phenomenon of workhardening of a deformed material. The description, as indicated in the title, concerns the work-hardening curve.

\section{CRITICAL ANALYSIS OF EXISTENT DESCRIPTIONS OF THE WORK- HARDENING}

It is worth starting this analysis with an information on the primary actions concerning the description of the phenomenon of plastic deformations. According to the literature [5], the attempts to work out the theory of the plastic deformations were begun back in the years 1868-1875 by H. Tresca, B. Saint-Venant, and M. Lévy. After the first steps, there was the 40-year period of discontunuation, until the following development with the works of $\mathrm{T}$. Karman, and A. Haar (1909), and R. Mises (1913) continued. Those works were the impulse to carry out more intensive research in the period after the 1st World War.

They were the beginning studies on the description of the phenomenon of plastic deformations. In 1959 the work [5] appeared, summing up the theory state on plasticity, presenting the rules and technical applications. From this work the first examples of the description of the work-hardening curve were excerpted to put it herewith under a critical analysis.

The attention has been drawn to two, discussed below, methods of the analytical presentation of the curve showing the dependence between the tensile stress $\sigma$ and the relative deformation $\varepsilon$, for the metals revealing the flow/strengthening. In the first method, the continuous curve has been presented approximately by two rectilinear segments, substituting the smooth transient curve with a sharp inflexion/break in the point of the coordinate assumed to be the yield point, which is the initial flow stress $\sigma_{0}$ (Fig. 1).

The first rectilinear branch of the plot refers to the elastic range and forms the angle equaled $\operatorname{arctg} E$ with the axis $\varepsilon$, with $E$ being the constant of the longitudinal elasticity of a material. The second rectilinear branch of the plot (Fig. 1), presenting an idealized strengthening area, forms the angle equaled $\operatorname{arctg} B$, with the axis $\varepsilon$ described by the equation

$$
\sigma=\sigma_{0}+B\left(\varepsilon-\frac{\sigma_{0}}{E}\right)=A+B \varepsilon
$$




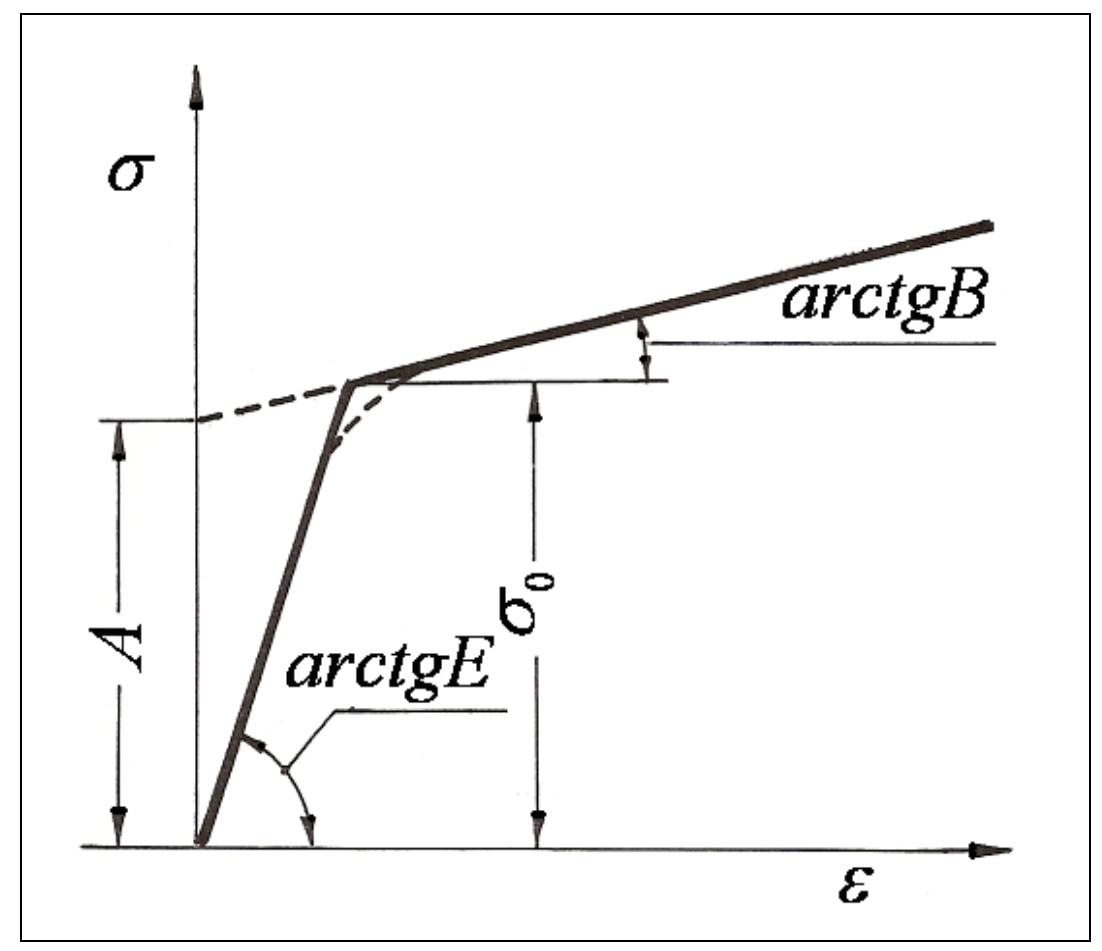

Fig. 1. Curve of the dependence between the stress and strain, presented approximately by two rectilinear segments [5].

where

$$
A=\sigma_{0}\left(1-\frac{B}{E}\right)
$$

A similar approximation is sometimes used by substituting a short part of the curve $\sigma-\varepsilon$ in the strengthening area by its tangent, with the tangent of inclination angle named the tangent modulus.

In the second approximation, a power term of type

$$
\sigma=k \varepsilon^{m}
$$

is used, where $k$ and $m$ (strengthening exponent) are the two characteristic constants of a metal, determined from the condition of the best conformity of dependence (3) with the curve determined experimentally.

That equation refers only to the area of strengthening (Fig. 2), with the beginning determining the initial flow stress $\sigma_{0}$ and the corresponding relative deformation $\varepsilon_{0}$.

That is the end of description of the work-hardening curve presented and excerpted from the literature [5]. Provided illustrations are original in character; no innovations have been introduced. 


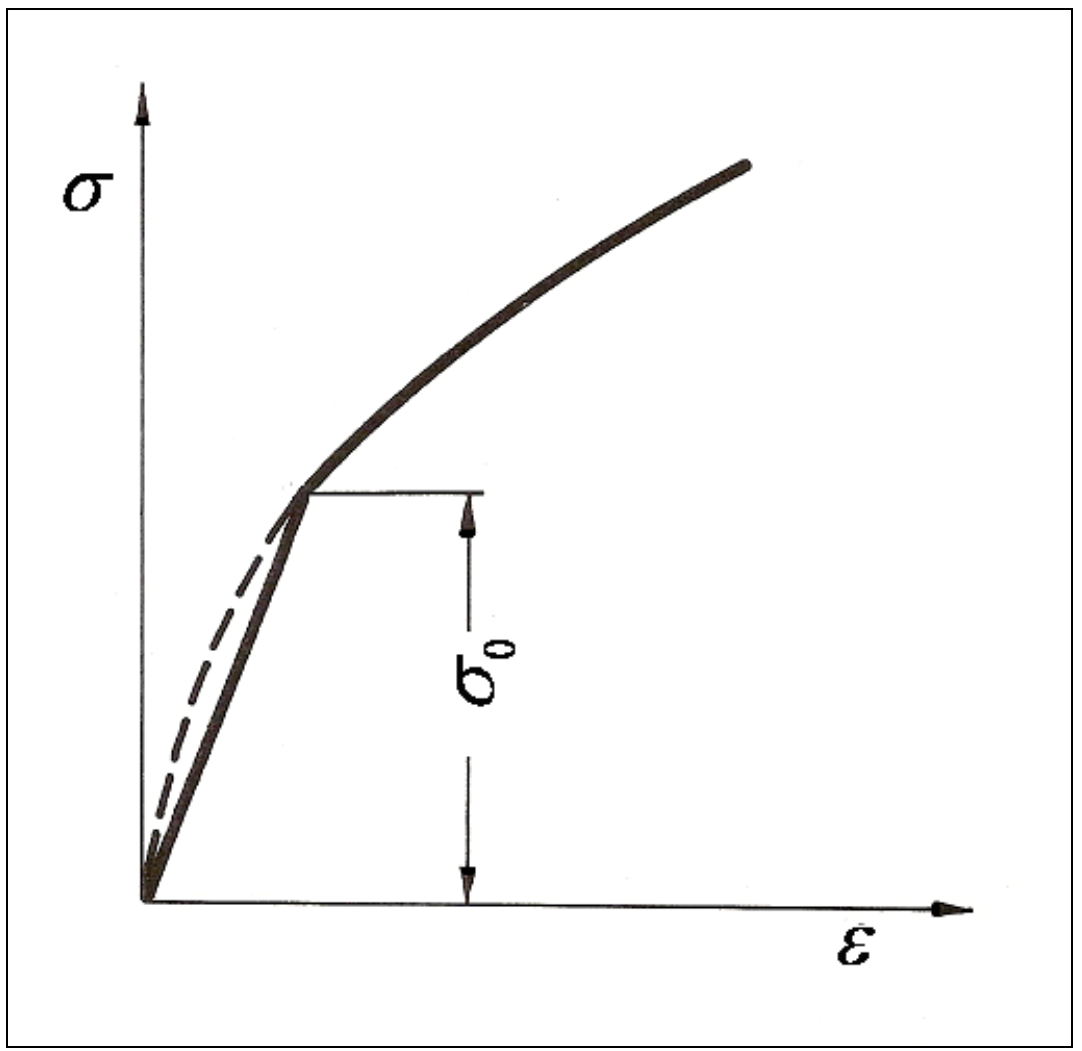

Fig. 2. Curve of the dependence between the stress and strain, determined in the plastic zone by a power expression [5].

Let us begin the critical analysis of the described matter. At the beginning it is worth noting the lack of the right-handed limit of the both presented descriptions; none of them has an end and suggests the phenomenon will last for an infinitely long time. Therefore those descriptions have inadequate character; there is no physical sense with them, because it is obvious the material strengthening/hardening phenomenon has its end (however, the descriptions indicate the beginning of the considered phenomenon).

Not only lack of the right-handed limit proves of the inadequacy of both presented models. The flaw is also in the simplified courses of the work-hardening curves; moreover, their descriptions have only empirical, statistic-experimental character, with no physical sense, in fact. In the frameworks of this analysis it is worth developing the power description of the work-hardening curve, regarding just the initial parameters of the phenomenon, i.e. $\sigma_{0}$ and $\varepsilon_{0}$. Therefore (Fig. 3)

$$
\sigma=\sigma_{0}+k\left(\varepsilon-\varepsilon_{0}\right)^{m}
$$

with $\varepsilon \geq \varepsilon_{0}$.

Furthermore, the dependence $\sigma=f(\varepsilon)$ is determined unilaterally. That means the description has its beginning determined univocally, by means of the magnitudes $\sigma_{0}$ and $\varepsilon_{0}$, and further continuation of this description has an open character. 


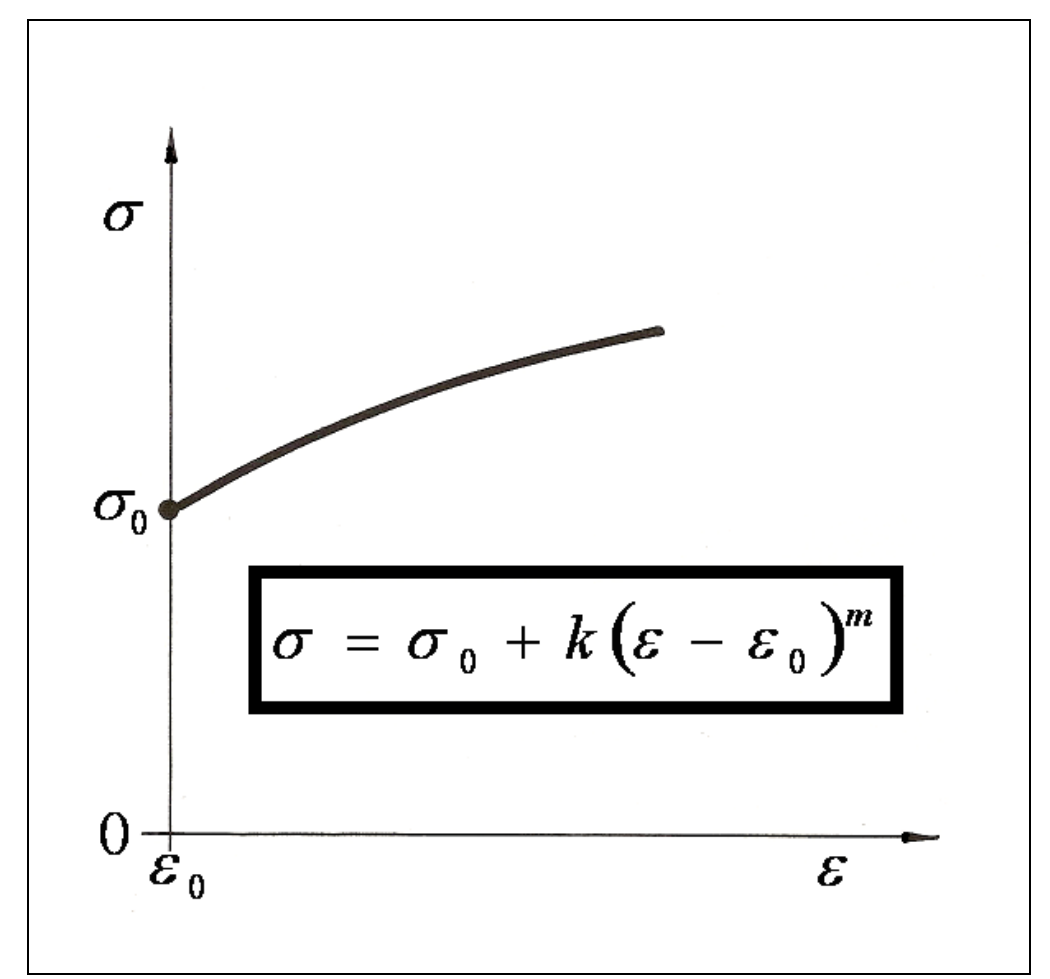

Fig. 3. Unilaterally determined dependence of the stresses on relative deformations.

That is not the end of the attempts to describe the work-hardening curve; the work [6], being a synthesis of the works carried out on request in the frameworks of a project, provides the following equation

$$
\sigma_{p}=C\left(\varepsilon_{0}+\varepsilon\right)^{n}
$$

To determine the constants $C, \varepsilon_{0}$, and $n$ of the three-parameter equation of this curve it is necessary to determine the coordinates experimentally, at least of three of its points. And the solution of such a system is rendered difficult because one of the unknowns is in the power exponent. It is worth looking at this equation and analysing it, first of all concerning the physical sense. A graphic picture of the described work-hardening curve (Fig. 4) will allow for better noticing the degree of its adequacy.

One may notice, the curve crosses the axis of the coordinates in the point $\sigma_{p}^{0}$, determined from the equation (5) by substituting to it $\varepsilon=0$; that is

$$
\sigma_{p}^{0}=C\left(\varepsilon_{0}\right)^{n}
$$

The stress $\sigma_{p}$ is zero for $\varepsilon=-\varepsilon_{0}$, that is for a deformation with absolutely no physical sense. The entry with the curve description into the second quarter of the assumed coordination system is a methodic error because that description loses the nature of the physical sense. The phenomena may be described only in the first quarter as only in this quarter the values of the dependent and independent variables are positive. 


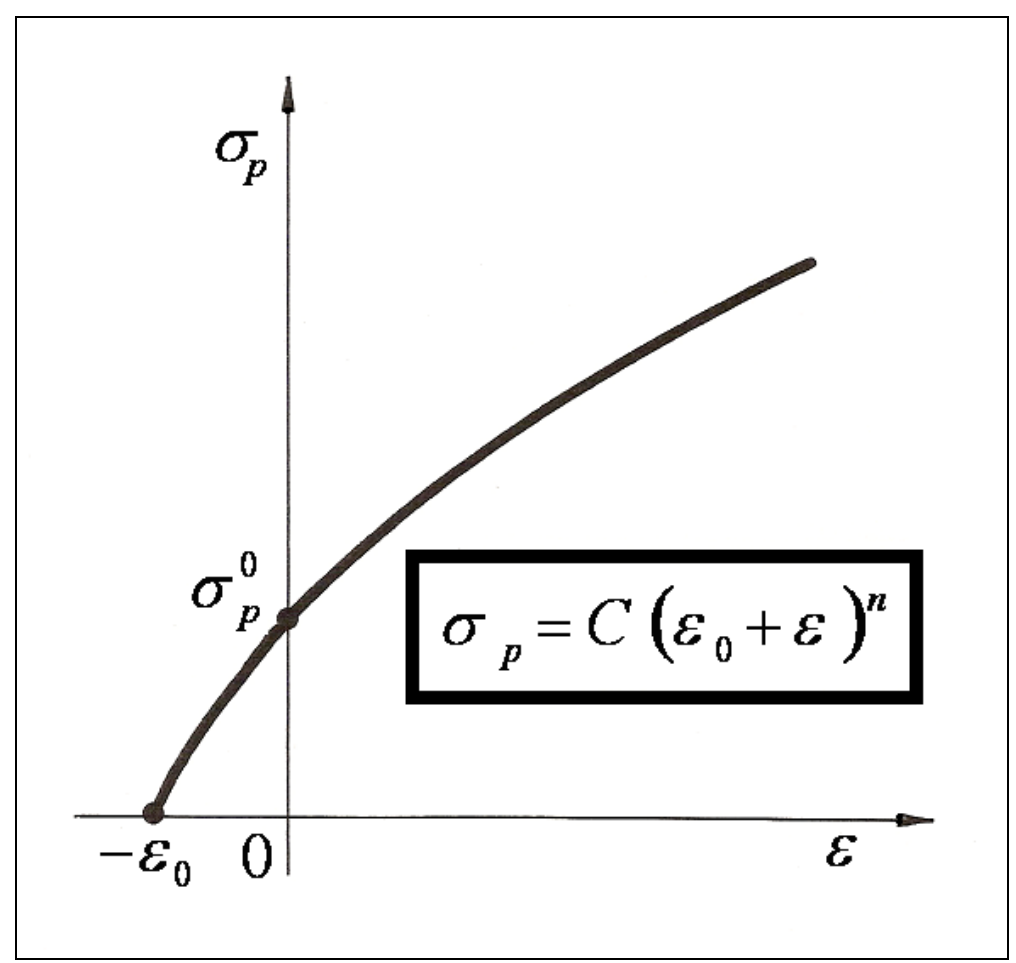

Fig. 4. Description and graphical illustration of the plastic work-hardening curve, acc. to [6].

Apart from that the essential flaw of the description of the work-hardening curve, it has been commonly used. It may be found, for instance, in the literature [7], where the study results of the mechanical properties of a laser joint of sheets of the car bodies, have been presented. That description is in the work [8], where among others, the connection of deformation of the cut material cross-section with its ability to the yield strain [9-11], has been indicated. The coefficient of hardening has been assumed as the measure of this ability with the power exponent of the dependence (5).

In these description actions it is sometimes assumed that the studied material has negligibly small initial (proper) stresses, that is $\sigma_{p}^{0}=0$, and then

$$
\sigma_{p}=C \varepsilon^{n}
$$

That way it was treated in the work [12], presenting the quantitative evaluation of the process of aluminium compression at elevated temperatures.

Presented herewith the description of the material hardening evidently indicates that the problem has not been solved adequately, with numerous cognitive gaps. First of all they lack any physical sense. In this situation there is a need or necessity to elaborate such a description with a prospected physical sense.

The description of this phenomenon should be started from the source; this source is the general differential equation, being the analytical reference to any phenomena of a quantum character (non-continuous, cascade-like). The equation of this type is contained in many previous Authors' works [1,4,13-15].

In this presented work the potential description source has been introduced to the description of phenomenon of the material hardening/strengthening. Furthermore, the 
mentioned general equation has been presented following the course of its detailed solution; then the way of using of the obtained analytical model of the considered technological system has been explained.

\section{ADEQUATE DESCRIPTION OF THE PLASTIC HARDENING CURVE}

At the source of this cognitive way of all physical phenomena, which occur with a variable rate/intensity or velocity between the neighbouring energetic states, there is a general differential description, namely:

$$
d Z= \pm \frac{\partial Z}{\partial N} d N
$$

where: $d Z$ - total differential of the magnitudes being the dependent variables, $d N$ - total differential of the magnitudes being the independent variables, $\partial Z / \partial N$ - partial derivative of the magnitudes dependent against independent ones. The signs $( \pm)$ are the algebraic operators fulfilling a determined function. The sign $(+)$ confirms the physical sense of a determined mathematic record, whereas the sign $(-)$ assigns a physical meaning to the record $[1,4]$.

The record of the equation (8), applied to the considered phenomenon, possesses the following configuration:

$$
d \sigma_{p}=-\frac{\partial \sigma_{p}}{\partial(\Delta \varepsilon)} d(\Delta \varepsilon)
$$

where, as can be seen, only negative algebraic operator is left. That means the changes of the plasticizing/yield stress are degressively rising, and the rate of these changes decreases with the deformation increase. The studies [16-18] confirm such a configuration of the hardening/strengthening curve. The image of dependence of the plasticizing stress $\sigma_{p}$ on the relative growth of deformation $\Delta \varepsilon$ is then the degressively rising the exponential curve (Fig. 5). This curve is the envelope of right-angled triangles, with the deformation leg being constant and equaled to the so called relative deformation constant $\Theta_{w}$, and the stress leg varied respectively, decreasing with the shifting of triangle in the direction of the deformation axis. The curve description of the dependence $\sigma_{p}=f(\Delta \varepsilon)$ requires first the creation of a determined network of the variable potential fields. They are stress and deformation potential fields, situated in the stress and deformation directions, respectively. At the very beginning of the stress direction there is the stress stable potential field $(S P F)_{\sigma}^{0}$. At the height of place, where the phenomenon of the material strengthening begins, there is the initial stress unstable potential field $(A P F)_{\sigma}^{0}$ situated. In the place of the completion of this phenomenon one may notice the final stress unstable potential field $(A P F)_{\sigma}^{1}$. At the top there is the stress nominal potential field $(N P F)_{\sigma}$ situated. On this field the right-angled triangle with the deformation leg is moving. 


\section{$\sigma_{p}=\sigma_{p}^{0}+2\left(\sigma_{p}^{1}-\sigma_{p}^{0}\right)\left(1-e^{-\frac{\varepsilon-\varepsilon_{0}}{\Theta_{w}}}\right)$}

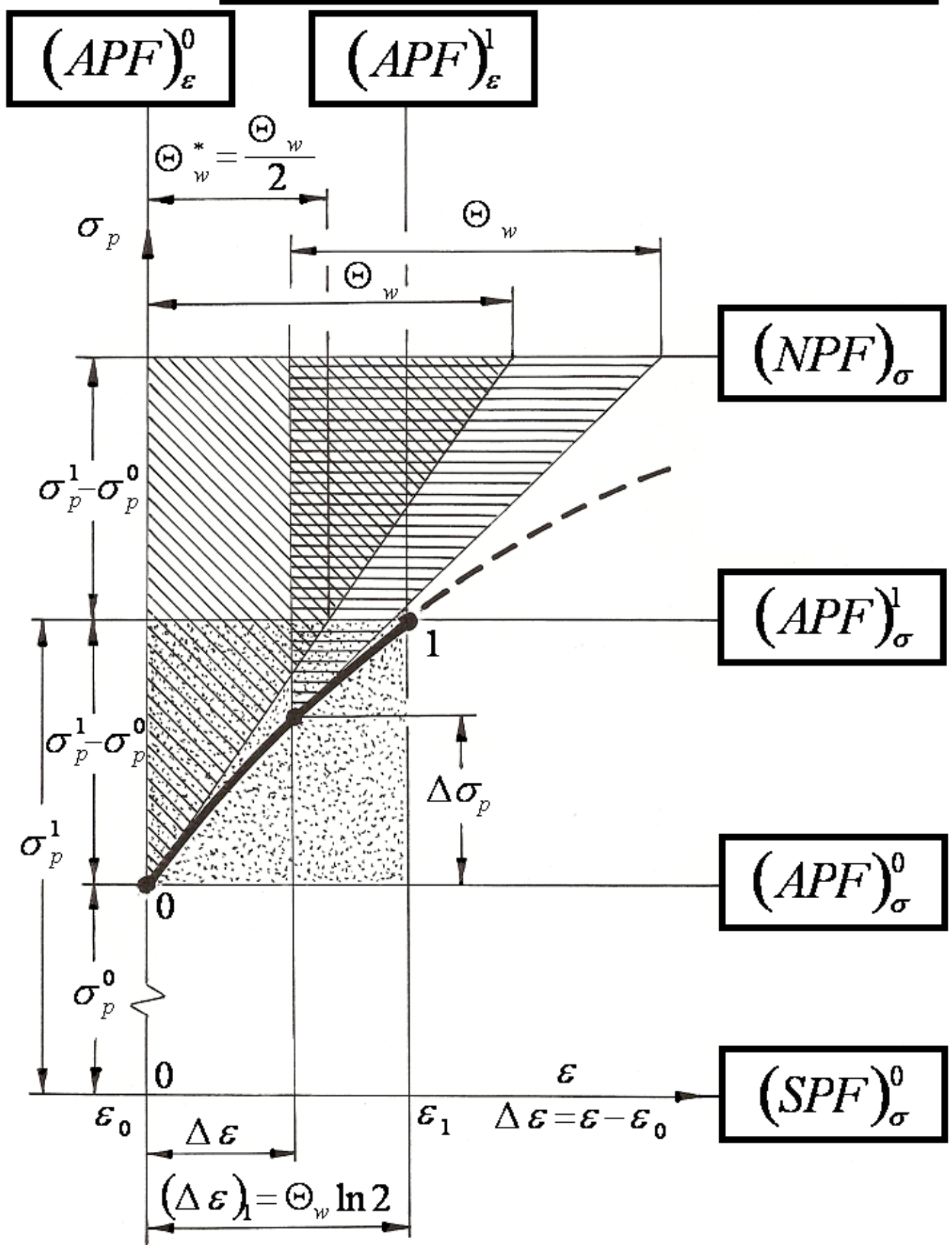

Fig. 5. Illustration of the plastic strengthening curve creation schematic. 
In the deformation direction, at the height of the initial deformation $\varepsilon_{0}$, there is the initial deformation unstable potential field $(A P F)_{\varepsilon}^{0}$; then, at the height of the final deformation $\varepsilon$, there is the final/terminal deformation unstable potential field $(A P F)_{\varepsilon}^{1}$ situated.

It is worth noticing that the initial phenomenon point (the moment of its beginning) is placed on the cross-section of the fields $(A P F)_{\varepsilon}^{0}$ and $(A P F)_{\sigma}^{0}$. The final point of the considered phenomenon is situated on the cross-section of the fields $(A P F)_{\varepsilon}^{1}$ and $(A P F)_{\sigma}^{1}$. The mentioned fields form the deformation-stress space (dotted area), with the enforcement curve (continuous line). That is a real and proper space; an improper space, forming the auxiliary space, adheres to it. Both these spaces (proper and improper ones) possess the same equal lengths in the stress direction, equaling the stresses difference $\sigma_{p}^{1}$ and $\sigma_{p}^{0}$.

That auxiliary design is quite necessary as it allows to describe the real course of workhardening curve and explains that the described phenomenon has also its end. Furthermore, in the improper space (dashed line), the only fiction course takes place, tending towards the horizontal asymptote which is the stress potential field $(N P F)_{\sigma}$.

Now one may begin integrating the equation (9), marking the limits of the integrals from the total differentials. That means

$$
\int_{\sigma_{p}^{0}+\Delta \sigma_{p}}^{\sigma_{p}^{1}+\sigma_{p}^{1}-\sigma_{p}^{0}} d \sigma=-\frac{\partial \sigma}{\partial \varepsilon} \int_{\Delta \varepsilon}^{\Delta \varepsilon+\Theta_{w}} d(\Delta \varepsilon)
$$

and then

$$
2\left(\sigma_{p}^{1}-\sigma_{p}^{0}\right)-\Delta \sigma_{p}=-\frac{d \sigma}{d(\Delta \varepsilon)} \Theta_{w}
$$

or

$$
\frac{d \sigma}{2\left(\sigma_{p}^{1}-\sigma_{p}^{0}\right)-\Delta \sigma_{p}}=-\frac{1}{\Theta_{w}} d(\Delta \varepsilon)
$$

One may notice, the partial derivative has been substituted by the ratio of the total differentials. It could be done that way because the total differentials have been clearly determined by introducing the limits to their integrals. result

Furthermore, by integrating both sides of the equation (12), one obtains the following

$$
\ln \left[2\left(\sigma_{p}^{1}-\sigma_{p}^{0}\right)-\Delta \sigma_{p}\right]=-\frac{1}{\Theta_{w}} \Delta \varepsilon+C^{*}
$$


or

$$
2\left(\sigma_{p}^{1}-\sigma_{p}^{0}\right)-\Delta \sigma_{p}=e^{-\frac{\Delta \varepsilon}{\Theta_{w}}+C^{*}}=e^{C^{*}} \cdot e^{-\frac{\Delta \varepsilon}{\Theta_{w}}}=C e^{-\frac{\Delta \varepsilon}{\Theta_{w}}}
$$

After regarding that for $\Delta \varepsilon=0$ the magnitude $\Delta \sigma_{p}=0$, one obtains

$$
C=2\left(\sigma_{p}^{1}-\sigma_{p}^{0}\right)
$$

and after substituting (15) to (14)

$$
\Delta \sigma_{p}=2\left(\sigma_{p}^{1}-\sigma_{p}^{0}\right) \cdot\left(1-e^{-\frac{\Delta \varepsilon}{\Theta_{w}}}\right)
$$

or

$$
\sigma_{p}-\sigma_{p}^{0}=2\left(\sigma_{p}^{1}-\sigma_{p}^{0}\right) \cdot\left(1-e^{-\frac{\varepsilon-\varepsilon_{0}}{\Theta_{w}}}\right)
$$

and finally

$$
\sigma_{p}=\sigma_{p}^{0}+2\left(\sigma_{p}^{1}-\sigma_{p}^{0}\right) \cdot\left(1-e^{-\frac{\varepsilon-\varepsilon_{0}}{\Theta_{w}}}\right)
$$

One may now determine the second coordinate of the point 1 , that is the deformation $(\Delta \varepsilon)_{1}$. It is obtained through the introducing the stresses $\sigma_{p}^{1}$ and the mentioned so marked deformation to the equation (18). Therefore

$$
(\Delta \varepsilon)_{1}=\varepsilon_{1}-\varepsilon_{0}=\Theta_{w} \ln 2
$$

As can be seen, the formula (18) is a physical formula with all its constant magnitudes $\left(\sigma_{p}^{0}, \sigma_{p}^{1}, \varepsilon_{0}, \Theta_{w}\right)$ being the physical magnitudes, having determined contents and units. One may create further derivative magnitudes, by determining simply the derivatives of these stresses against the deformation. Let us stay with the first derivative, being the measure of the intensity, rate of increase of the plastisizing/yield stresses, or the ability to the hardening/ strengthening. Therefore 


$$
U=\frac{d \sigma_{p}}{d \sigma(\Delta \varepsilon)}=\frac{2\left(\sigma_{p}^{1}-\sigma_{p}^{0}\right)}{\Theta_{w}} e^{-\frac{\varepsilon-\varepsilon_{0}}{\Theta_{w}}}
$$

That ability in the initial point, for $\Delta \varepsilon=0$, is determined by the following formula:

$$
U_{0}=\left(\frac{d U}{d(\Delta \varepsilon)}\right)_{\Delta \varepsilon=0}=\frac{2\left(\sigma_{p}^{1}-\sigma_{p}^{0}\right)}{\Theta_{w}}
$$

Naturally, the derived formulae (18), (20), and (21) have a general character and are concerned with different types of the material deformation. If the material is extended, then the distinct plasticity limit $R_{e}$ (or the apparent one $R_{0,2}$ ), fulfills the role of the initial stresses $\sigma_{p}^{0}$, whereas the tensile strength $R_{m}$ corresponds with the final stresses $\sigma_{p}^{1}$. At this material deformation manner the deformation $\varepsilon$ is the relative elongation, that is the ratio of absolute increment of the sample length $\Delta l$ to its initial length $l_{0}$, then

$$
\varepsilon=\frac{\Delta l}{l_{0}}
$$

\section{DEPENDENCE OF THE YIELD FORCE ON THE ABSOLUTE DEFORMATION INCREMENT}

The problem of the description of the work-hardening curve may be developed, and it is worth explaining, e.g. on the material tensile. Presentation of this curve in other way, as the picture of the dependence of the yield force $F_{p}$ on the increment $\delta l$ of the absolute deformation $\Delta l$, will allow to obtain some other, important magnitudes. It is about the yield rigidity, yield work, and yield/plastic potentials.

The dependence (18) may be presented in the mentioned system $F_{p}-\delta l$ in the following way:

$$
F_{p}=F_{e}+2\left(F_{m}-F_{e}\right) \cdot\left(1-e^{-\frac{\Delta l-(\Delta l)_{0}}{\Theta_{b}}}\right)
$$

as

$$
\delta l=\Delta l-(\Delta l)_{0}
$$

with: $F_{e}$ - sharp yield point, $F_{m}$ - tensile strength force, $\Theta_{b}$ - absolute deformation constant. A graphic picture of that dependence (Fig. 6) is also the degressive exponential curve. 


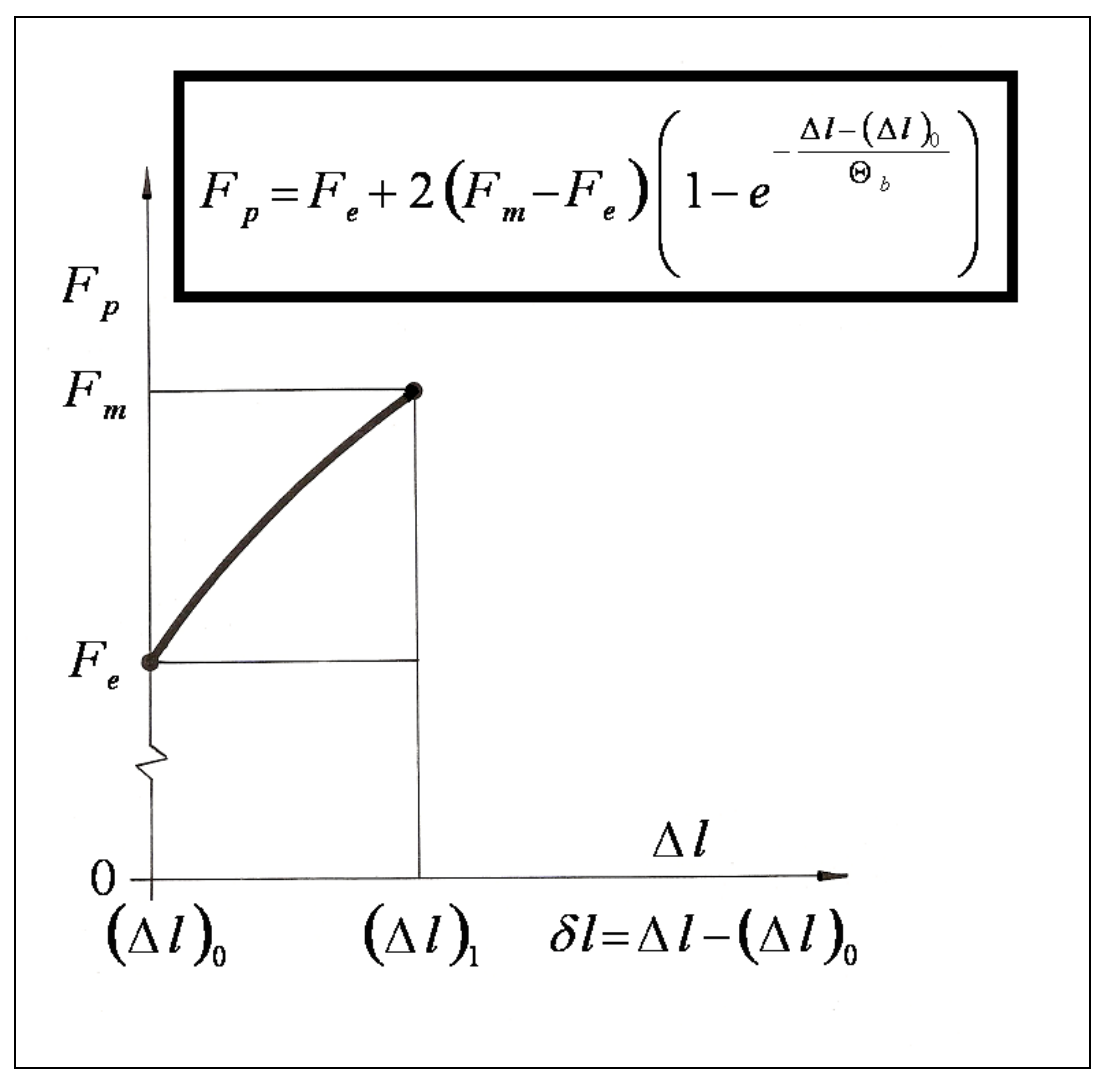

Fig. 6. Plot of dependence of the yield force on the absolute deformation increment.

One may obtain further characteristics of the hardening/strengthening phenomenon. The derivative of the yield force against the absolute increment of the material extension, i.e. $\delta l$, is the coefficient of its plastic rigidity. Therefore

$$
k_{p}=\frac{d F_{p}}{d(\delta l)}=\frac{2\left(F_{m}-F_{e}\right)}{\Theta_{b}} e^{-\frac{\Delta l=(\Delta l)_{0}}{\Theta_{b}}}
$$

That coefficient in the point of the beginning of the hardening/strengthening phenomenon, that is for $\delta l=0$, has the following analytic form

$$
k_{p}^{0}=\left(\frac{d F_{p}}{d(\delta l)}\right)_{\delta l l-0}=\frac{2\left(F_{m}-F_{e}\right)}{\Theta_{b}}
$$

Now one may determine the yield work, which is simply the definite integral of the function $F_{p}(\delta l)$, that is 


$$
L_{p}=\int_{0}^{(\Delta l)_{1}-(\Delta l)_{0}} F_{p}(\delta l)
$$

Therefore

$$
\begin{aligned}
& L_{p}=\int\left[F_{e}+2\left(F_{m}-F_{e}\right) \cdot\left(1-e^{-\frac{\delta l l}{\Theta_{b}}}\right]\right] \cdot d(\delta l)= \\
& =\left[F_{e} \delta l+2\left(F_{m}-F_{e}\right) \cdot \delta l+2 \Theta_{b}\left(F_{m}-F_{e}\right) e^{-\frac{\delta l}{\Theta_{b}}}\right]_{0}^{(\Delta l)_{1}-(\Delta l)_{0}}= \\
& =F_{e}\left[(\Delta l)_{1}-(\Delta l)_{0}\right]+2\left(F_{m}-F_{e}\right)\left[(\Delta l)_{1}-(\Delta l)_{0}\right]-2 \Theta_{b}\left(F_{m}-F_{e}\right) \\
& \left(1-e^{-\frac{(\Delta l)_{1}-(\Delta l)_{0}}{\Theta_{b}}}\right.
\end{aligned}
$$

It is time now to define and mark the phenomenon of the hardening/strengthening of these plastic potentials on the characteristics. They are two types of the potentials: initial, and the final one. Of course, these potentials are the energy measures: the initial yield energy, and final yield energy, respectively. Thus, as it results, the energy itself is not a physical magnitude; indeed its measure (potential) has the nature of such a magnitude, as the physical one.

Then, what is the yield energy? It is a mental magnitude, defined by the mind and introduced to describe a determined real system. Until now the notion of the energy (not only the plastic one) has not been clearly defined. The literature [19-23] proves that. It is defined mainly as the ability to perform work, with the identification of the energy with work, putting both these magnitudes (mental and physical magnitudes) before/in-front-of and after/behind the sign of the identity.

It is worth explaining this notion. Generally speaking, the energy of a solid is its readiness to perform a nominal/rated work over it, or the ability to perform the work by it. Thus, it is about the apparent magnitudes, which should be introduced to characterize the quantum (energetic) states of a material body.

In reference to the considered phenomenon it is about plastic/yield energy and nominal/rated yield/plastic work. Therefore (according to this energy definition, the plastic/yield one) in the direction of the system loading (Fig. 7), at the beginning of this activity, it is about the plastic/yield energy of the body as its readiness to perform the nominal/rated yield work over it. In the direction of the system de-loading (at the end of its loading) it is about the plastic/yield energy of the body, being the ability to perform such a work with it. 


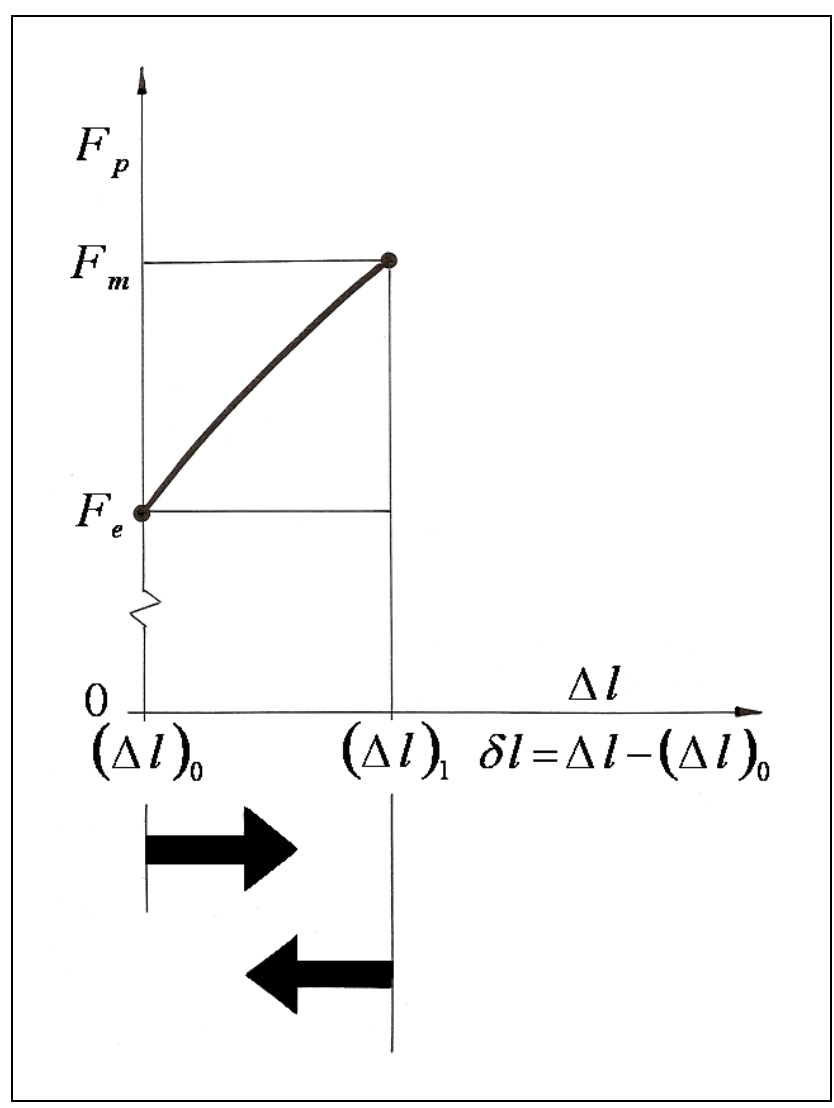

Fig. 7. Directions of the solid deformation determining detailed definitions of its yield energy.

Therefore the initial yield potential $V_{p}^{0}$ determines the following term:

$$
V_{p}^{0}=F_{p}^{0} \cdot\left[(\Delta l)_{1}-(\Delta l)_{0}\right]
$$

The final yield potential $V_{p}^{1}$ is expressed by the following formula:

$$
V_{p}^{1}=F_{p}^{1} \cdot\left[(\Delta l)_{1}-(\Delta l)_{0}\right]
$$

with the terminal forces $\left(F_{p}^{0}, F_{p}^{1}\right)$ being simultaneously, under the energetic approach to the hardening/strengthening phenomenon, the intensities of the respective deformation fields.

A graphic approach to both of these potentials, specifically of the yield work, with the analytical record reflected by the formula (28), has been presented in Fig. 8. The areas filled with horizontal lines form a kind of the energetic bands. 


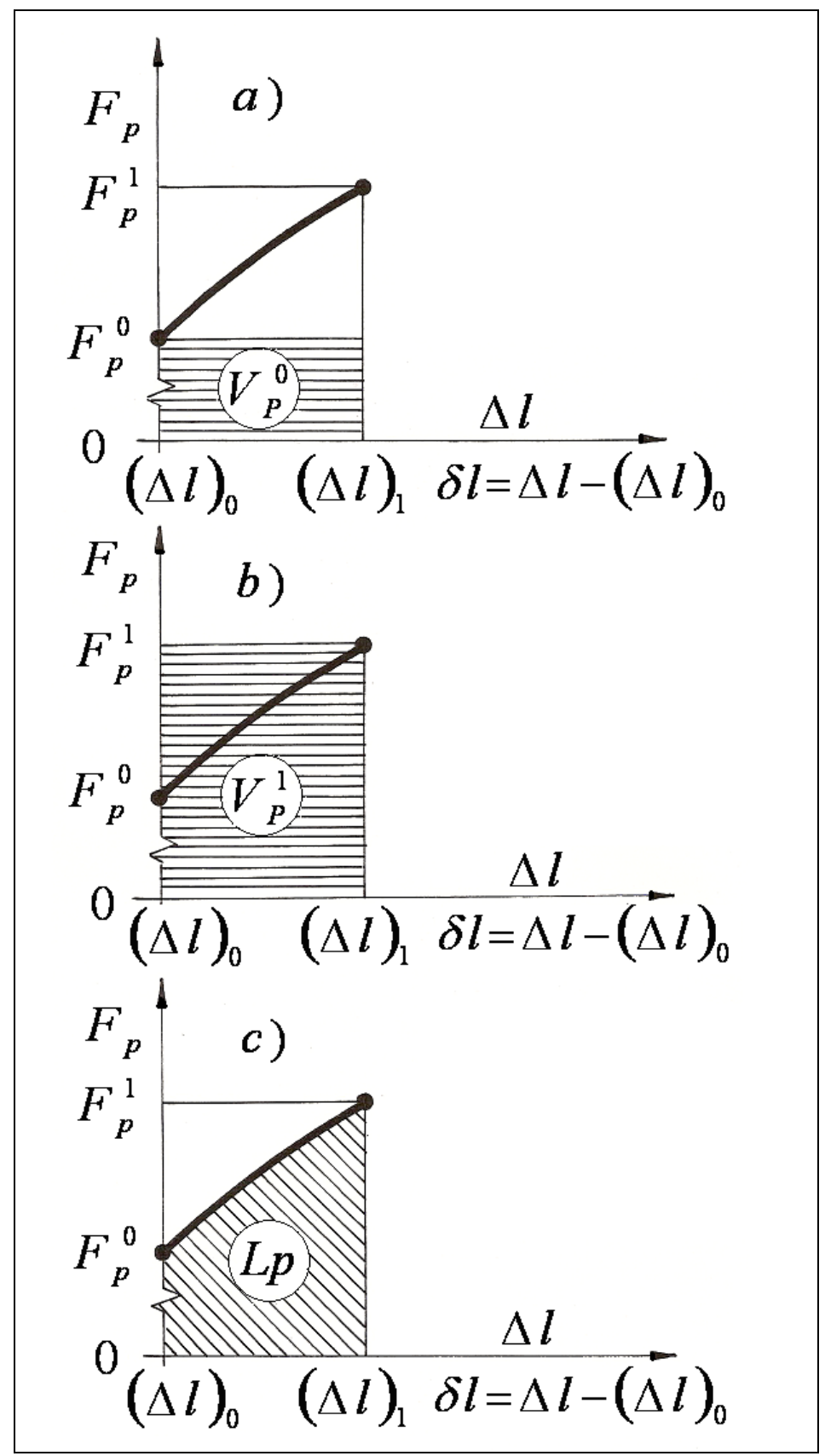

Fig. 8. Initial plastic/yield potential (a), final plastic/yield potential (b), and the yield/plastic work (c).

\section{CONCLUSION}

In the frameworks of the conclusion it is worth turning the attention on the cognitive core of the paper, containing quite a new approach to the description of the particular phenomena, here the phenomenon of the material hardening/strengthening. Presented herewith the adequate description of this phenomenon, possesses a physical sense, covering the whole phenomenon, with entire its nature. And this nature, as indicated and proved, has a quantum, jump, non-continuous character. The continuity has been marked only between the neighbouring states, where both the beginning and the end of the considered phenomenon take place. 
The adequate description of the material hardening/strengthening has become the starting point to create further characteristics of the phenomenon. Thus also the plastic/yield rigidity and the yield work have been described. The quantum nature of the phenomenon has been characterized, by introducing entirely explained the energy notion, and the yield energy in particular.

It is worth also, on the basis of the considered phenomenon, putting stress on the scientific terminology. A variety of magnitudes, coming from the four classification groups have appeared; they are mental, sensorial, physical, and material ones. The yield energy is a mental magnitude, whereas its measure, i.e. the potential, has the nature of physical magnitude. The plastic rigidity is the sensorial magnitude, whereas the coefficient of plastic rigidity, the physical magnitude. The yield work is naturally also the physical magnitude. The last magnitudes, the material ones, do not require explanations.

These four groups of the magnitudes may be presented in the form of a pyramid (Fig. 9). At the top of the pyramid there are the most important, mental magnitudes M; just below, the sensorial ones S; then, the physical ones P; and at the bottom, the material ones M. Such a presentation of all these magnitudes, also the graphic one, has been presented in the literature [24-25]. The particular exemplary magnitudes, creating the cognitive structure of this work, have been referred to the particular groups of this pyramid in Fig. 9.

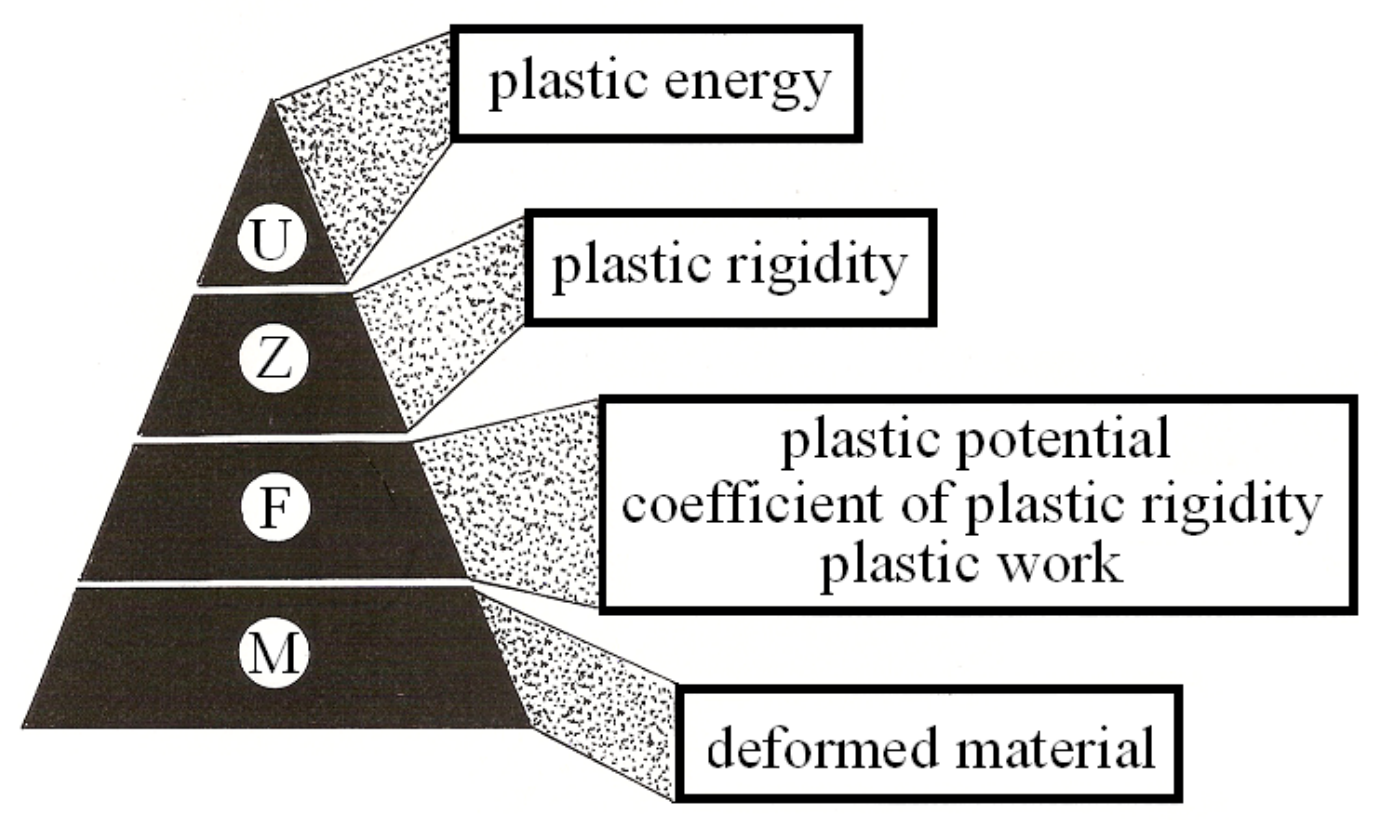

Fig. 9. Pyramidal structure of the magnitudes and the relevant examples.

Presented herewith the method of description of the reality may be applied to more complex characteristics of the materials, deformed in a different way. It is possible to analytically describe, for instance, the entire curve of the material tension, all its fragments which have the determined state limits, proving of that between them, to occur different phenomena of a quantum character. 


\section{NOMENCLATURE}

$\varepsilon$ - relative deformation

$\Delta l$ - absolute deformation

$\sigma_{p}-$ yield stress

$d(\Delta \varepsilon)$ - total differential of the relative deformation increment

$d \sigma_{p}$ - total differential of the yield stress

$\frac{\partial \sigma_{p}}{\partial(\Delta \varepsilon)}$ - partial derivative of the yield stress against the relative deformation increment

$k_{p}-$ coefficient of the plastic rigidity

$\Theta_{w}-$ relative deformation constant

$\Theta_{b}-$ absolute deformation constant

$U$ - hardening ability

$F_{p}$ - yield force

$F_{e}$ - sharp yield point

$F$ - tensile strength force

$L_{p}-$ plastic/yield work

$V_{p}^{0}-$ initial plastic/yield potential

$V_{p}^{1}$ - final plastic/yield potential

$(S P F)_{\sigma}^{0}-$ stress stable potential field

$(A P F)_{\sigma}^{0}-$ initial stress unstable potential field

$(A P F)_{\sigma}^{1}$ - final stress unstable potential field

$(N P F)_{\sigma}$ - stress nominal potential field

$(A P F)_{\varepsilon}^{0}$ - initial deformation unstable potential field

$(A P F)_{\varepsilon}^{1}$ - final deformation unstable potential field

\section{References}

[1] Zdzisław Pluta, Tadeusz Hryniewicz, Int. J. Adv. Manuf. Technol. 51 (2010) 35-43, DOI: $10.1007 / \mathrm{s} 00170-010-2595-5$.

[2] Zdzisław Pluta, Tadeusz Hryniewicz, Int. J. Adv. Manuf. Technol. 42 (2009) 515-522.

[3] Zdzisław Pluta, Tadeusz Hryniewicz, Int. J. Adv. Manuf. Technol. 43 (2009) 440-448.

[4] Zdzisław Pluta, Fundamentals of surface smoothing by elastic grinding wheels (in Polish), Publishing Koszalin University of Technology Koszalin, 2007.

[5] O. Hoffman, G. Sachs, Introduction to the theory of plasticity (in Polish), PWT, Warszawa, 1959 (transl. from English).

[6] Z. Marciniak, Mechanik 4 (1983) 199-207.

[7] J. Lisok, K. Mutwil, Przegląd Mechaniczny 10 (2009) 27-30. 
[8] S. Dzidowski S., Mechanik 12 (1988) 557-559.

[9] S. Dzidowski, Mechanik 5 (1984) 249-251.

[10] S. Dzidowski, J. Mechanical Working Technology 12 (1986) 297-306.

[11] S. Dzidowski S., Rozprawy Inżynierskie PAN IPPT, 2 (1984) 267-274.

[12] F. Seyna, A. Rudnik, Przeglad Mechaniczny 15 (1977) 534-536.

[13] Zdzisław Pluta, Tadeusz Hryniewicz, Intern. J. Adv. Mater. Technol. 62(5) (2012) 529-542, DOI: 10.1007/s00170-011-3813-5.

[14] Zdzisław Pluta, Tadeusz Hryniewicz, J. Modern Physics 3(7) (2012) 625-635; DOI: $10.4236 / j m p .2012 .37086$.

[15] Zdzisław Pluta, Tadeusz Hryniewicz, J. Modern Physics 3(8) (2012) 793-802; DOI: $10.4236 / j m p .2012 .38104$.

[16] F. Grosman, J. Łukowski, Mechanik 4 (1977) 172-175.

[17] E. Milczarek, Mechanik 2 (1987) 63-64.

[18] A. Niechajowicz, A. Tabota, L. Nakonieczny, Mechanik 12 (1988) 577-579.

[19] P. Atkins, Galileo finger (in Polish), Publishing Dom Wydawniczy REBIS, Poznań, 2006, (transl. from English), 1st Edition.

[20] P. M. Harman, Energy, force, and matter: The conceptual development of nineteenth century physics, Cambridge University Press, 1982.

[21] B. Lightman (Ed.), Victorian science in context, University of Chicago Press, 1977.

[22] C. Smith, The science of energy: A cultural history of energy physics in Victorian Britain, Athlone Press, London 1988.

[23] J. Barbour, The discovery of dynamics, Oxford University Press, 2001.

[24] Zdzisław Pluta, Forum Akademickie 3 (1999) 58-60.

[25] Tadeusz Hryniewicz, Zdzisław Pluta, Proc. of the $8^{\text {th }}$ World Conference on Continuing Engineering Education, Theme A: Knowledge, Skills and Competency, May 12-16, 2001, Toronto, Ontario, Canada, pp. 25-31. 